\section{Tratamento da crise aguda de asma em países emergentes}

Prezado Editor,

Apreciamos com especial interesse o estudo de Vilarinho et al. ${ }^{1}$ deste periódico, mesmo porque estamos em fase de implementação desta forma de tratamento da crise de asma em uma unidade pública de atendimento de emergências, onde são realizados mais de 33.000 atendimentos pediátricos/ano, sendo a crise aguda de asma responsável por $13 \%$ dessas consultas ${ }^{2}$.

Os inaladores pressurizados dosimetrados com ou sem espaçadores têm sido extensivamente estudados na população pediátrica e são recomendados em consenso no tratamento da asma aguda e/ou persistente ${ }^{3}$. Entretanto, desconhecemos em Curitiba algum serviço de emergência, público ou privado, que tenha instituído o seu uso como rotina.

Após Zar et al. ${ }^{4}$ fomentar a idéia de produzir espaçadores artesanais (garrafas plásticas de refrigerante) para substituir os industrializados, com a conseqüente redução de custos e eficácia semelhante, outros estudos foram realizados demonstrando seus benefícios, inclusive um deles realizado em Juiz de Fora, Brasil ${ }^{5}$.

Isso torna este assunto de importância ímpar para os chamados países emergentes, onde investigamos alternativas de tratamento que reduzam os custos e comprovem eficácia. Com a intenção de familiarizar e verificar como esses dispositivos funcionariam para a população de sua região, Vilarinho et al. realizaram um estudo bem intencionado, porém alguns aspectos éticos e metodológicos devem ser advertidos:

1) A Resolução 196/96 do Conselho Nacional de Saúde (órgão que rege as normas para pesquisa em seres humanos no Brasil) não permite o uso de um grupo exclusivamente placebo nessa situação, devido ao princípio da não-maleficência ao paciente. Contudo, tornar este estudo duplo-cego controlado com placebo aumentaria a confiabilidade de seus resultados ${ }^{6}$.

2) A falta de um consentimento livre e esclarecido assinado pelos pais e/ou guardiões legais pode trazer complicações legais futuras, principalmente por tratar-se de um grupo especial, com crianças com idade inferior a 18 anos. A autorização verbal e o questionamento em relação à aceitação ou não do dispositivo foram sempre direciona- das aos pais. Em algum momento foram questionadas as crianças, mesmo que somente aquelas com capacidade para responder tal pergunta?

3) O planejamento prévio de uma pesquisa reduz a possibilidade de viés em seus resultados, devendo-se realizar um projeto piloto para a estimativa do tamanho da amostra necessária para que o estudo tenha um poder estatístico adequado $^{7}$. Procuramos hoje, em pesquisa, a Medicina Baseada em Evidências ${ }^{8}$, e não apenas o que mais se aproxima da "vida real", como valoriza a autora.

4) Os grupos eram homogêneos em relação à gravidade das crises. Por que oxigênio foi oferecido apenas para o grupo NEB? Poderia o grupo IDE obter uma melhor performance com o uso de oxigênio inalado após a administração do salbutamol? Seriam os gastos no tratamento do grupo NEB superestimados devido ao uso de oxigênio?

5) O escore de Wood-Downes para a classificação da gravidade da crise de dispnéia e sibilância apresenta pontuação que varia entre 0 e 2 para cada parâmetro avaliado, e o mesmo foi validado por Paes et al. ${ }^{9}$. Pode esta adaptação realizada para o estudo ser valorizada?

Devemos sempre continuar investigando alternativas eficazes e de baixo custo para o tratamento da asma aguda, porém devemos ser cautelosos com novas idéias no anseio de popularizá-las, divulgando esses conceitos com metodologia científica adequada. Corremos o risco de tornar essas brilhantes descobertas uma forma "tupiniquim" de medicar, pois tanto em pesquisa como na vida real estamos sujeitos a processos éticos e civis.

\section{Referências}

1. Vilarinho LCS, Mendes CMC, Souza LS. Inalador dosimetrado em espaçador artesanal versus nebulizador no tratamento de crise de sibilância na infância. J Pediatr (Rio J). 2003;79:403-12.

2. Chong Neto HJ, Silva DC, Rosário NA, Lara J. Frequency of emergency room visits for acute asthma attacks in inner-city children. Abstracts of XVIII World Allergy Organization Congress; 2003 September 7-12; Vancouver, Canada: J World Allergy Org. 2003; Suppl 1:92-3.

3. Sociedade Brasileira de Pneumologia e Tisiologia, Sociedade Brasileira de Alergia e Imunopatologia, Sociedade Brasileira de Pediatria e Sociedade Brasileira de Clínica Médica. III Consenso Brasileiro no Manejo da Asma. J Pneumol. 2002; 28 Suppl 1:S4-28.

4. Zar HJ, Brown G, Donson H, Brathwaite N, Mann MD, Weinberg EG. Home-made spacers for bronchodilator therapy in children with acute asthma: a randomized trial. Lancet. 1999;354:979-82.

5. Duarte M, Camargos P. Efficacy and safety of a home-made nonvalved spacer for bronchodilator therapy in acute asthma. Acta Paediatr. 2002;91:909-13.

6. Kirshner B. Methodological standards for assessing therapeutic equivalence. J Clin Epidemiol. 1991;44:839-49. 
7. Orwin RG. A fail-safe N for effect size. J Educ Stat. 1983;8:157-9.

8. Greenhalgh T. Evidence-based medicine. In: Hall M, Dwyer D, Lewis T, editors. GP Training Handbook. 3rd ed. Oxford: Blackwell Scientific; 1998.

9. Paes R, Percebo A, Naspitz C, Solé D. Escores de gravidade para episódios agudos de dispnéia e sibilância em crianças: validação dos escores de Wood-Downes e Ben-Zvi. Rev Bras Alerg Imunopatol. 2002;25:122-30.

\section{Herberto J. Chong Neto}

Especialista em Pediatria, SBP, em Alergia e Imunologia Clínica, SBAI, e Mestrando em Medicina. Grupo de Pesquisa em Patologia Pediátrica, Pontifícia Universidade Católica do Paraná (PUC-PR).

\section{Débora C. Silva}

Especialista em Pediatria, SBP, em Alergia e Imunologia Clínica, SBAI, e Mestranda em Medicina. Grupo de Pesquisa em Patologia Pediátrica, Universidade Federal do Paraná.

\section{Lúcia Noronha}

Professora adjunta, Departamento de Patologia Clínica, Hospital das Clínicas, UFPR e PUC-PR. Doutora em Medicina. Grupo de Pesquisa em Patologia Pediátrica, PUC-PR e UFPR.

\section{Resposta dos autores}

\section{Prezado Editor,}

O tratamento da asma em crianças na sua manifestação aguda e crônica nos serviços públicos brasileiros ainda é deficitário. Existem plenas evidências de que a terapia inalatória é a melhor em termos de efetividade e redução dos efeitos colaterais ${ }^{1}$. Urge que se viabilizem formas éticas de facilitação do tratamento, principalmente através dos inaladores dosimetrados com espaçadores, para que haja melhora na deposição da droga nos pulmões.

A realização do estudo publicado no Jornal de Pediatria, "Inalador dosimetrado com espaçador artesanal versus nebulizador no tratamento da crise de sibilância na criança" foi concebida pela necessidade de se validar uma prática já habitual no Hospital Universitário da UFBa, que consistia na utilização de espaçadores artesanais para possibilitar o tratamento de crianças com aerossol dosimetrado, em especial as muito pequenas ou com dificuldades de adaptação aos nebulizadores. A troca dos nebulizadores pelo aerossol dosimetrado foi motivada pela necessidade de se reduzirem custos hospitalares com o uso de oxigênio. Além disso, as vantagens operacionais já eram conhecidas através de estudos realizados com copos de café e diferentes tipos de inaladores com ou sem válvulas, de vários tamanhos e formatos, selados ou não ${ }^{2-5}$. Pela relevância do tema, este veio a se tornar-se objeto de dissertação de mestrado de um dos autores (L.C.S. Vilarinho), com orientação de outro autor (L.S. FreitasSouza), e por isso foi exaustivamente discutido na sua metodologia e aspectos éticos. Um estudo piloto foi realizado, não para cálculo de tamanho amostral (pois estudos pilotos não se prestam a essa determinação), porém tão somente para averiguação da validade interna. Como não havia possibilidade do cálculo prévio do tamanho da amostra, optamos pela também tecnicamente correta amostra de conveniência, termo que não é tão familiar aos não-especialistas em estatística e que indica que o estudo é interrompido no momento em que chegamos nos limites de recursos de qualquer natureza. E também por isso, o cálculo de poder foi feito a posteriori, não tendo atingido os desejáveis $80 \%$, indicando que teria sido necessário estudar uma amostra muito maior para se alcançar o poder de $80 \%$.

Não procede comentar sobre o uso de placebo neste desenho de estudo pois não estamos comparando drogas e sim dispositivos inalatórios. Além do mais, isso seria completamente antiético, de acordo com a Resolução do Conselho Nacional de Saúde. Como deixar alguém sem tratamento, já sabidamente eficaz, principalmente se isso acarreta risco de vida?

Nosso consentimento informado verbal foi em relação a podermos usar os dados da criança em trabalho científico, já que os procedimentos terapêuticos realizados - nebulização ou uso de aerossol dosimetrado com espaçador artesanal - já fazem parte da rotina do nosso prontoatendimento desde 1996.

O termo "vida real" utilizado diz respeito à inclusão de todas as idades de 0 a 12 anos, refletindo a população que é atendida no nosso pronto-atendimento, em vez de termos selecionado uma faixa etária restrita, a ponto de só podermos utilizar nossos resultados em casos especiais. Isso faz parte também da metodologia deliberadamente programada. A nossa escolha acarreta possíveis variáveis confundidoras (como a inclusão de lactentes sibilantes não-asmáticos, que poderiam ter fraca resposta ao broncodilatador), porém tem o aspecto positivo de permitir avaliar o resultado das duas formas de tratamento no nosso pronto-atendimento como um todo, e responder à questão: é vantajoso usar o aerossol dosimetrado com espaçador dosimetrado no pronto-atendimento em crianças de qualquer idade?

O oxigênio foi oferecido no grupo NEB apenas para gerar o aerossol no nebulizador de jato. Poderia ter sido usado ar comprimido ao invés. Porém nosso CPPHO ainda não dispunha de ar comprimido: as nebulizações são rotineiramente realizadas com $\mathrm{O}_{2}$. Se forem observados, na tabela 3 do trabalho, os valores de oximetria de pulso (média, valores mínimos e máximos e mediana), poder-se-á concluir que, apesar de não ter usado $\mathrm{O}_{2}$, o grupo IDE teve valores de saturação de hemoglobina iguais ou superiores aos do grupo NEB, e, a julgar pelos valores basais (TO), nenhum dos grupos teria necessidade de uso de $\mathrm{O}_{2}$.

O custo do tratamento com nebulização fica elevado por causa do $\mathrm{O}_{2}$, que é necessário para gerar o aerossol. Os custos de instalação e uso de ar comprimido são bem mais baixos, cerca de $1 / 3$ do custo com oxigênio, porém inviáveis para nosso serviço na época do estudo. Os aparelhos compressores portáteis, em geral, não geram aerossol adequado: as partículas são grandes e com grande variabilidade de diâmetro; portanto, não devem ser utilizados.

A tabela de Wood-Downes utiliza parâmetros hemogasométricos e, assim, não é adequada para uso no início do tratamento da crise, momento em que não se realiza hemo- 
gasometria (por não ser necessária) e sim apenas oximetria (quando possível). Por essa razão, construímos a tabela utilizada neste trabalho, contendo parâmetros clínicos que indicam o comprometimento da função pulmonar (alguns dos quais estão na tabela de Wood \& Downes) e a oximetria, também já validada como parâmetro de estudo da gravidade da asma6.

O trabalho foi desenvolvido com metodologia científica adequada e detalhadamente referida em Material e métodos. Conforme relatamos na Discussão, o estudo é preliminar, porque usamos uma amostra de conveniência, sem cálculo amostral prévio, justamente pela ausência, na literatura, de trabalhos com metodologia semelhante que pudéssemos utilizar como base para o cálculo do tamanho amostral; agora, nosso estudo pode vir a servir de base para esse cálculo para outros trabalhos caso haja interesse em se estender essa linha de pesquisa.

Expressamos nosso protesto veemente com relação ao uso da expressão "tupiniquim" em tom depreciativo e o firme ponto de vista de que nós, brasileiros, precisamos deixar o colonialismo cultural e científico em que vivemos e investir em busca de nossa própria identidade, no desenvolvimento da nossa capacidade de criar conhecimento em sintonia com nossas necessidades e na coragem de expressá-lo.

\section{Leda Solano de Freitas Souza}

Professora Adjunta de Pediatria - Faculdade de Medicina da Universidade Federal da Bahia. Chefe do Setor de Pneumologia do Centro Pediátrico Prof. Hosannah de Oliveira - Universidade Federal da Bahia.

\section{Liana Consuelo Santana Vilarinho}

Mestrado em Pediatria (UFBA). Professora colaboradora do Dep. de Pediatria da Faculdade de Medicina da UFBA.

\section{Carlos Maurício Cardeal Mendes}

Doutorado em Saúde Coletiva - concentração em Epidemiologia. Médico estatista do Instituto de Saúde Coletiva (UFBA) e Professor de Saúde Coletiva e Estatística da Escola Baiana de Medicina.

\section{Referências}

1. Sociedade Brasileira de Alergia e Imunopatologia, Sociedade Brasileira de Pediatria e Sociedade Brasileira de Pneumologia e Tisiologia. II Consenso Brasileiro no Manejo da Asma. J Pneumol. 1998;24:171-276.

2. Everard $M L$, Clark AR, Milner AD. Drug delivery from holding chambers with attached facemask. Arch Dis Child. 1992;67: 580-5.

3. Keeley D. Large volume plastic spacers in asthma should be used more. BMJ. 1992;305:598-9.

4. Barry PW, O'Callaghan C. Inhalational drug delivery from seven different spacer devices. Thorax. 1996;51:835-40.

5. Hindle $M$, Chrystyn $\mathrm{H}$. Relative bioavailability of salbutamol to the lung following inhalation using metered dose inhalation methods and spacer devices. Thorax. 1994;49:549-53.

6. Cubells CL, Garcia JJ, Torrico PG, Mora MLS, Diaz EC. Utilidad de un sistema de puntuación clínico y la pulsioximetria $\left(\mathrm{Sat}_{2}\right)$ en la valoración de la gravedad de las crisis de asma. Ann Esp Pediatr. 1996;44:429-32.

\section{Tabagismo durante a gravidez: um problema maior do que se imagina}

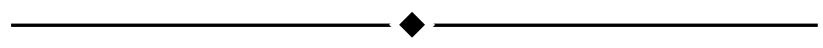

O tabagismo interfere na função reprodutora das muIheres no período pré-concepção, na evolução da gravidez e na lactação ${ }^{1}$. Uma vez que as gestantes estão em contato regular com os profissionais de saúde (acompanhamento pré-natal), as campanhas antitabagismo normalmente surtem um ótimo efeito durante esse período. No entanto, para que esses esforços sejam bem-sucedidos, devemos estar atentos às seguintes considerações:

1) Enquanto que o uso de drogas ilícitas durante a gravidez vem recebendo grande atenção nas últimas duas décadas, muito pouca atenção tem sido dada às conseqüências do uso de "drogas sociais", como fumo, álcool e cafeína, que são certamente as drogas mais comumente consumidas durante a gravidez.

2) Enquanto que os efeitos nocivos da cocaína, anfetaminas e opióides nas mães e nos fetos são mais acentuados e fáceis de identificar, o tabagismo, o etilismo e o consumo de cafeína são normalmente mais sutis e mais difíceis de diagnosticar ${ }^{2}$. Conseqüentemente, o uso recreacional do fumo durante a gravidez pode permanecer despercebido, afetando significativamente a evolução da gravidez e a lactação.

3) Aproximadamente $80 \%$ das mulheres que fumam antes da gravidez continuam a fumar enquanto grávidas ${ }^{2}$. o baixo consumo de cigarros antes da gravidez é o melhor fator preditivo para a interrupção do tabagismo durante a gravidez.

4) A maioria das pacientes com histórico de uso de drogas durante a gravidez (incluindo fumo) nega seu uso quando entrevistada por médicos atendentes, obstetras e/ou neonatologistas ${ }^{3}$.

5) Os fatores de risco que sugerem o uso de fumo durante a gravidez incluem ausência de acompanhamento prénatal, complicações respiratórias e histórico de parto prematuro.

É necessário, portanto, que tenhamos uma grande desconfiança quanto ao uso do fumo (bem como de outras drogas sociais e ilícitas) durante a gravidez. Também devemos adotar uma postura não-crítica em relação a cada paciente.

\section{Referências}

1. Mello PR, Pinto GR, Botelho C. The influence of smoking on fertility, pregnancy and lactation. J Pediatr (Rio J). 2001;77: 257-64.

2. Kuczkowski KM. Tobacco and ethanol use in pregnancy: implications for obstetric and anesthetic management. The Female Patient. 2003;28:16-22. 